\title{
Small-Angle Neutron Scattering of Soot Formed in Laminar Premixed Ethylene Flames
}

Hai Wang

University of Delaware

Bin Zhao

University of Delaware

Barbara E. Wyslouzil

Worcester Polytechnic Institute, barbaraw@wpi.edu

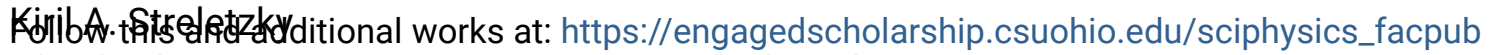
I'nveland State University, K.STRELETZKY@csuohio.edu

Part of the Physics Commons

How does access to this work benefit you? Let us know!

\section{Publisher's Statement}

NOTICE: this is the author's version of a work that was accepted for publication in Proceedings of the Combustion Institute. Changes resulting from the publishing process, such as peer review, editing, corrections, structural formatting, and other quality control mechanisms may not be reflected in this document. Changes may have been made to this work since it was submitted for publication. A definitive version was subsequently published in Proceedings of the Combustion Institute, 29, 2, 2002 DOI\#10.1016/S1540-7489(02)80335-2

\section{Repository Citation}

Wang, Hai; Zhao, Bin; Wyslouzil, Barbara E.; and Streletzky, Kiril A., "Small-Angle Neutron Scattering of Soot Formed in Laminar Premixed Ethylene Flames" (2002). Physics Faculty Publications. 263.

https://engagedscholarship.csuohio.edu/sciphysics_facpub/263

This Article is brought to you for free and open access by the Physics Department at EngagedScholarship@CSU. It has been accepted for inclusion in Physics Faculty Publications by an authorized administrator of EngagedScholarship@CSU. For more information, please contact library.es@csuohio.edu. 


\title{
Spectral Time Moment Analysis of Microgel Deswelling Effect of the Heating Rate
}

\author{
KIRIL A. STRELETZKY, JOHN T. MCKENNA
}

\section{INTRODUCTION}

Temperature sensitive microgels are nanoparticles synthesized via crosslinking of amphiphilic polymer chains with easily achievable volume phase transition. ${ }^{1-3}$ Convenient systems for microgel synthesis include amphiphilic macromolecules that are soluble in water at room temperature with hydrogen bonds formed between the chain's hydrophilic groups and water. As temperature increases above the critical temperature $T_{\mathrm{c}}$, water becomes a poor solvent due to weakening of the hydrogen bonds. The resulting inability of hydrophilic chain-water interactions to counter hydrophobic chain-chain interactions leads to macromolecular self-association into metastable aggregates and separation of HPC solution into two phases.

The polymer's sensitivity to temperature and other environmental factors that make the solvent poor (such as $\mathrm{pH}$, electric field, salt concentration) is inherited by microgels and results in controllable and reversible volume phase transition of nanoparticles. ${ }^{1,3}$ For temperature sensitive systems, heating of the microgel sample above $T_{\mathrm{c}}$ causes nanoparticles to deswell. During the deswelling process, microgels expel water due to hydrophobic interactions. The result of this 
transition is an effective "shrinking" of microgel volume by as much as a couple orders of magnitude. ${ }^{4}$ The transition is reversible and microgels return to their original size by absorbing water if the solution temperature is lowered below $T_{\mathrm{c}}$. A similar external stimuli-sensitive volume phase transition occurs in bulk polymer gels (known as hydrogels). However, microgel nanoparticles exhibit a much faster response to environmental changes due to their small size. ${ }^{4}$ The controllable volume phase transition of microgels leads to numerous potential applications such as: particle emulsifiers in food and cosmetics, ${ }^{5,6}$ shear-thinning dispersions in surface coatings and paints, ${ }^{2}$ drug loading, and delivery vesicles in pharmaceutical industry, ${ }^{1,7}$ oil permeability modifiers, ${ }^{8}$ and environmentally responsive devices in bio-nanotechnology. ${ }^{9,10}$ In addition, microgels present a convenient model system for studying: phase transition in polymer solutions, ${ }^{11}$ variety of polymer diffusion problems ranging from chains to polymer aggregate dynamics in solutions,${ }^{1}$ and the dynamics of concentrated colloidal suspensions. ${ }^{3}$

The polymer studied in this work is hydroxypropylcellulose (HPC), which is an electrically neutral, semi-stiff, rod-like polysaccharide with a persistence length of $100 .{ }^{12,13}$ HPC is soluble in a wide range of solvents including water, but has an easily achievable pseudotheta conditions at a critical temperature of $T_{\mathrm{c}}=41{ }^{\circ} \mathrm{C} \cdot{ }^{13-16} \mathrm{At}$ room temperature $\left(T \leq T_{\mathrm{c}}\right)$ water is a good solvent to HPC chains and solutions are homogeneous. Above $T_{\mathrm{c}}$ hydrophobic interactions are favorable and water becomes a poor solvent for HPC chains. This forces the solution to separate into two phases; one of which is made from poorly soluble metastable aggregates of self-associated chains. ${ }^{16,17}$ The phase transition is reversible: lowering solution temperature below $T_{\mathrm{c}}$ brings back the isotropic, one phase system. ${ }^{18}$ HPC:water solutions also exhibit a lyotropic phase transition which starts at ambient temperature in the concentration range $360-420 \mathrm{~g} / \mathrm{L} .{ }^{19}$

Because of its convenient physical properties and wide accessibility HPC has been used extensively in studies of polymer dynamics. ${ }^{13,15,18,20-23}$ The polymer has numerous pharmaceutical and food industry applications as it is FDA-approved for food and clinical consumption..$^{24,25}$ In particular, HPC polymer is used as a tablet binder ${ }^{26,27}$ due to its high cohesiveness, binding strength, hardness, and low friability. HPC hydrogel is popular in sustained release matrix schemes and is used as a bio-adhesive for trans-mucosal drug administration. ${ }^{24}$ The polymer is also used as a thickener and stabilizer ${ }^{24,27}$ to maintain and build up viscosity of pharmaceutical liquid.

HPC chains can be chemically cross-linked into stable microgel nanoparticles above the critical temperature $T_{\mathrm{c}}{ }_{\mathrm{c}}^{4,16}$ or even at room temperature by effectively lowering $T_{\mathrm{c}}$ with salt addition ${ }^{17,28}$ consequently lowering the magnitude of steric interactions. In fact, the synthesized microgels undergo a reversible temperature dependent volume-phase transition at the temperature $T_{\mathrm{v}}$ which is somewhat higher than $T_{\mathrm{c}}$. The transition above $T_{\mathrm{v}}$ brings deswelling of microgel nanoparticles with a significant release of solvent that can be equal to many times the weight of microgel itself. The phase transition can be reversed by the temperature decrease, which results in swelling of microgels due to absorption of the solvent. The volume phase transition of microgels is caused by the hydrophobic-hydrophilic temperature dependence of the HPC polymer itself. However, while uncrosslinked HPC solutions form metastable particles above $T_{\mathrm{c}}$, HPC microgel nanoparticles are stable both above and below $T_{\mathrm{v}}$ because they are held by a cross-linker.

The structural and behavioral versatility of HPC microgel particles makes them good candidates for applications in a variety of targeted drug delivery schemes. ${ }^{29,30}$ Many controllable parameters including polymer and salt concentration, cross-linking density, chemical make up, and synthesis protocol affect microgel size, loading capacity and swelling/deswelling ability. ${ }^{17}$ Other parameters of microgel systems may also be adjusted to effectively load medicines into the structure, target diseased tissue (e.g. cancer), and release the payload upon predetermined environmental conditions (such as high temperature or $\mathrm{pH}$ ). For example, microgel can undergo self-induced volume phase transition at lower $T_{\mathrm{v}}$ if salt is added to the existent microgel solution. In fact, ref. 4 reports lowering $T_{\mathrm{v}}$ to $39{ }^{\circ} \mathrm{C}$ upon achieving the physiological ionic strength $(0.9$ wt $\%$ of 0.15 $\mathrm{mol} / \mathrm{L}$ ) in microgel solutions. The relatively high value of $T_{\mathrm{v}}$ for HPC microgels gives them an advantage over widely studied PNiPAM microgels as their volume phase transition temperature (even at the physiological ionic strength) is not too close to the temperature of healthy tissue.

The synthesis techniques for HPC microgels have been studied extensively.,16,17,29 $\mathrm{Lu}$ et al. ${ }^{4}$ synthesized microgels via an emulsion method by crosslinking $1 \mathrm{MDa} \mathrm{HPC}$ polymer chains in 
aqueous surfactant solutions above the critical temperature $T_{\mathrm{c}}=41{ }^{\circ} \mathrm{C}$. Gao et al. ${ }^{16}$ induced selfassociation of microgels via an addition reaction of $100 \mathrm{kDa} \mathrm{HPC}$ and a crosslinker (no surfactant) in a basic environment $(\mathrm{pH}=12-13)$ above $T_{\mathrm{c}}$. Xia et al. ${ }^{17}$ formed $1 \mathrm{MDa}$ HPC microgels using the addition reaction of ref. 16 , but at an ambient temperature. This was achieved by lowering $T_{\mathrm{c}}$ from $41^{\circ}$ to $23^{\circ} \mathrm{C}$ with salt addition. Reference 29 used a free radical polymerization to create HPC microgels with degradable moieties.

The swelling/deswelling behavior of formed nanoparticles at the volume phase transition temperature has been studied by each of the aforementioned references in detail. References $4,16,17,29$ used dynamic light scattering (DLS) to deduce the size and shape of HPC microgels both above and below the transition. References 4,17,29 relied on the cumulant analysis ${ }^{31}$ of light scattering spectra $(S(q, t))$ to obtain the microgel's molecular weight average diffusion coefficient ( $D_{\text {ave }}$ ) and its distribution. Reference 16 reports obtaining $D_{\text {ave }}$ using both the cumulants and the Laplace inversion methods. ${ }^{32}$ The microgel's hydrodynamic radius was then calculated from $D_{\text {ave }}$ under the assumption that microgel particles are spheres with a narrow size distribution in a small-molecule solvent. While these assumptions might be acceptable for some microgel systems (for example PNi$\mathrm{PAM}^{1,3}$ ), careful analysis of the results from refs. $4,16,17$ and more recent findings ${ }^{28}$ on $1 \mathrm{MDa}$ HPC microgels (synthesized using the recipe of ref. 17) indicate highly nonexponential spectra and a wide bimodal size distribution. Under the conditions of substantially nonexponential spectra the initial slope of the DLS spectrum does not describe $S(q, t)$ completely and cumulants have a hard time weighting different contributions when calculating $D_{\text {ave }} \cdot{ }^{13,21}$ Therefore, the usage of cumulants in these systems is not advisable. In addition, refs. 16,17 relied only on the DLS data at the low scattering angles of $20^{\circ}$ and $30^{\circ}$, respectively. The low scattering angle data is the noisiest and would probe only large distance scales which unfairly neglects the smaller species. Indeed, ref. 4 studied microgels at angles of $90^{\circ}$ and $20^{\circ}$ and found that a decrease of an angle within this range brought a $20 \%$ increase in size and a significant narrowing of the size distribution. This result is an indication of the multi-modality in the prepared microgel solutions and the incorrectness of the assumption of the microgel's spherical nature. Another indication of the difficulty that cumulant analysis had in interpreting the spectra of ref.
17 is a reported high polydispersity index (1.31.5), which illustrates an excessive broadness of deduced size distribution. ${ }^{13}$

Reference 28 meticulously examined the highly nonexponential light scattering spectra of microgels synthesized using the recipe of ref. 17. Instead of relying on the cumulant analysis used by Xia et al., ${ }^{17}$ ref. 28 employed the spectral time moment analysis. ${ }^{23}$ The switch in the analysis approach was motivated by the apparent ${ }^{13,21,33}$ ambiguity of the cumulants method in the analysis of highly nonexponential spectra. In the method used by ref. 28 the spectra were fit to a sum of three stretched exponential modes and the time moments for each mode were found from decay distribution. Both temperature and angular dependences of the three spectral modes were carefully studied. The importance of an angular dependence of the scattering spectrum should not be downplayed as it provides for a direct test of diffusiveness in the observed microgel modes (even if there is only one mode) and, therefore, allows conclusions to be made about dominant microgel shape. The approach of ref. 28 revealed that the HPC microgel solutions (obtained under the recipe of ref. 17) yield at least three different contributions to overall size distribution: two particulate modes ("fast" and "intermediate"), which have properties of diffusive ("fast" mode) and almost diffusive ("intermediate" mode) particles, and a third ("slow") mode which has striking similarities with the slow mode of uncrosslinked HPC polymer chains. The Stokes-Einstein size estimates for the two diffusive modes gave apparent radii of $25-30 \mathrm{~nm}$ for the "fast" mode and 400-650 nm for the "intermediate" mode. The temperature dependence of the three spectral modes illustrates the complexity of the volume phase transition by showing the merging of the "fast" and "intermediate" modes above $T_{\mathrm{v}}$ and the shrinkage of the apparent particulate radius of the "intermediate" mode from $400-650 \mathrm{~nm}$ down to $100-150 \mathrm{~nm}$ as temperature was increased from $23^{\circ}$ to $45{ }^{\circ} \mathrm{C}$.

Although the interpretations of HPC microgel spectra differ somewhat between refs. 4, 16, 17 and ref. 28 , the effect of temperature increase on microgel deswelling has been described as "significant" by every aforementioned study. What has not been explored in detail (to author's knowledge) is the role of the heating history on the HPC microgel deswelling. Gao et al. ${ }^{16}$ reported that the formation of metastable HPC aggregates in uncrosslinked polymer solutions depends on "how the system is brought to that temperature." They 
found that the metastable HPC clusters formed under multi-step heating to $42{ }^{\circ} \mathrm{C}$ had an apparent hydrodynamic radius of about $210 \mathrm{~nm}$, while clusters formed under sudden heating from $25^{\circ}$ to $42{ }^{\circ} \mathrm{C}$ resulted in clusters with apparent radius of $135 \mathrm{~nm}$. The role of the heating rate on the phase separation of uncrosslinked, concentrated HPC solutions was studied in detail by Kuy et al. ${ }^{34}$ who found the phase separation to occur in accordance with the spinodal decomposition mechanism at very high heating rates (a few ${ }^{\circ} \mathrm{C} / \mathrm{min}$ ). The importance of the heating rate on thermally induced gelation of concentrated HPC solution was also reported by ref. 35 . They found no apparent gelation process present at a very slow heating rate. Therefore, one would expect for the heating history to have an important role in the volume phase transition of crosslinked HPC chain formations.

This article presents a time moments analysis of dynamic light scattering results on the HPC microgels subjected to different heating protocols. The focus of this article is on the effect of the rate of heating on different microgel modes observed both above and below the volume phase transition. The second section describes the experimental procedures used and the approach of the time moment analysis to characterize nontrivial DLS spectra. The third section of the manuscript presents the findings on the effect of the heating rate on microgel volume phase transition. Finally, the results are brought together for a summary in the fourth section.

\section{EXPERIMENTAL}

Dynamic light scattering spectroscopy was used to study the dynamics of microgel particles above and below the volume phase transition $T_{\mathrm{v}}$. In DLS, the intensity of the scattered light $I(q, t)$ is measured and recorded as intensity-intensity correlation function $g^{(2)}(q, t)$ which can be related to a field correlation function $g^{(1)}(q, t)$ via Siegert's relation:

$$
g^{(2)}(q, t)=A\left(g^{(1)}(q, t)\right)^{2}+B,
$$

where $A$ is the scattering amplitude, determined by various apparatus-dependent factors; $B$ is the time independent baseline of the spectrum; and $q$ is the magnitude of the scattering vector. Here, $q=\frac{4 \pi n}{\lambda_{0}} \sin \frac{\theta}{2}$ depends on the index of refraction of the medium, $n$; the wavelength of light in vacuum, $\lambda_{0}$; and the scattering angle, $\theta$. Most experiments were performed at the scattering angle $\theta=90^{\circ}$ corresponding to $q=2.30 \times 10^{-2} \mathrm{~nm}^{-1}$.

To obtain a distribution of relaxation rates in the studied system, spectra were analyzed by the line shape analysis ${ }^{22}$ on the level of $g^{(1)}(q, t)$ instead of relying on an Inverse Laplace Transform of $S(q, t)$ (e.g. CONTIN algorithm ${ }^{32}$ ). The nonlinear-least-squares simplex-based minimization procedure fitted each $g^{(1)}(q, t)$ repeatedly to sums of stretched exponentials with different initial parameters and/or number of modes, checking for RMS error and numerical stability of the fits. The procedure (described in detail in ref. 28) yielded the optimal functional form for the field correlation function as a sum of stretched exponentials:

$$
g^{(1)}(q, t)=\sum_{i=1}^{N} A_{i} \exp \left(-\theta_{i} t^{\beta_{i}}\right),
$$

here $A_{i}$ is a mode amplitude, $\theta_{i}$ is a decay pseudorate, $\beta_{i}$ is a stretching parameter, and $N$ is a number of modes. The sum of three stretched exponentials $(N=3)$ was found to provide the most reproducible, while not necessarily an unique, functional form for all attempted fits to a field correlation function. The fits to a sum of three stretched exponentials found small RMS fitting errors $\left(\leq 2 \times 10^{-4}\right)$ and were stable.

To understand the physical properties of the system, the method of spectral time moments ${ }^{22,23}$ was employed to analyze the obtained best fits to $g^{(1)}(q, t)$. Since $g^{(1)}(q, t)$ was fitted to a sum of three stretched exponentials, the properties of each mode had to be studied individually to identify physical processes corresponding to each mode. In fact, the spectral time moment analysis allows for direct calculation of the spectral time moments $M_{\mathrm{n}}$, which are the integral averages over the field correlation function. Because of the preferred functional form of the fits to $g^{(1)}(q, t)$ (the sum of stretched exponentials) the time moments for each mode can be found by integrating the fitting function analytically. In particular, zeroth time moments for each mode, $M_{0 i}$, can be calculated as:

$$
M_{0 i}=\int_{0}^{\infty} \mathrm{d} t \exp \left(-\theta_{i} t^{\beta_{i}}\right)=\gamma\left(1+1 / \beta_{i}\right) / \theta_{i}^{1 / \beta_{i}},
$$

here $\gamma(1+n)=n$ ! is the Gamma function denoted as $\gamma$ to distinguish it from a spectral decay rate $\Gamma$. 
For each exponential relaxation the mean relaxation time of a decay is equal to the zeroth time moment $M_{0 i}$. Therefore, one can deduce the mean decay rate $\left\langle\Gamma_{i}\right\rangle$ for each mode from $M_{0 i} / 0 ! \equiv\left\langle\Gamma_{i}^{-1}\right\rangle$. Also, a mean diffusion coefficient corresponding to each mode $D_{i}$ can be calculated from the corresponding $\left\langle\Gamma_{i}\right\rangle$ via $D_{i}=\left\langle\Gamma_{i}\right\rangle / q^{2}$.

This approach allows for calculation of the mean relaxation time and mean diffusion coefficient of each mode from an accurate fit of the field correlation function to the measured spectra without a direct need for perfect measurements of $g^{(2)}(q, t)$ at very large times as is often the case with CONTIN.

As was described in detail in ref. 28 , the two faster spectral modes of microgel spectra were found to exhibit the diffusive-like behavior $\left(\left\langle\Gamma_{i}\right\rangle \sim\right.$ $q^{2}$ each with a small intercept, if any). For these two modes we used the Stokes-Einstein equation:

$$
D=\frac{k_{\mathrm{B}} T}{6 \pi \eta R_{\mathrm{h}}}
$$

to estimate apparent hydrodynamic radii $R_{\mathrm{h} 1}$ and $R_{\mathrm{h} 2}$ that would correspond to the mean diffusion coefficients $D_{1}$ and $D_{2}$, respectively. In eq. $4, k_{\mathrm{B}}, T$, $\eta$ are the Boltzmann's constant, the absolute temperature of the solution, and the solvent viscosity. The third spectral mode was found to be independent of $q$ and was considered inappropriate for application of the Stokes-Einstein equation. Indeed, one should be very careful in using eq. 4 as it is derived for the case of dilute Brownian spheres in small-molecule, low-viscosity solvents. It has been shown theoretically ${ }^{36}$ and experimentally $^{33}$ that eq. 4 is not valid when a solution is concentrated enough to have a solvent viscosity very different from solution viscosity. Therefore, we considered this approach useful only for the two spectral modes ("fast" and "intermediate"), which showed diffusive-like behavior.

Spectra-Physics Stabilite $2017 \mathrm{Ar}^{+}$laser with vertically polarized light at $\lambda_{0}=514.5 \mathrm{~nm}$ and power output of 1-1.5 W was used for the experiments. The laser was coupled to a BI 200 SM photometer-goniometer (Brookhaven Instruments) and an ALV-5000 correlator.

Microgels were prepared by crosslinking chains of hydroxypropylcellulose (1 MDa nominal molecular weight, from Scientific Polymer Products) with divinyl sulfone (purchased from Aldrich). The solutions had a resulting polymer concentration of $0.05 \mathrm{wt} \%$. The synthesis followed the recipe of ref. 17 which is described in detail in ref. 28 .
The microgel solution was filtered through cellulose $1.2 \mu \mathrm{m}$ pore diameter filters (Osmonics) into pre-cleaned and dried cylindrical glass sample cells (with 10 cc volume). During experiments the cells were placed into a copper cell holder with a decalin-filled index-matching quartz vat. The entire cell assembly was maintained at a specific temperature $\left(23^{\circ} \leq T \leq 45{ }^{\circ} \mathrm{C}\right)$ within 0.1 ${ }^{\circ} \mathrm{C}$ with the help of a Thermo RTE-7 refrigerated bath/circulator.

Two different heating rates were used in microgel deswelling studies. Under the fast heating protocol the solution temperature was changed by a step of $5{ }^{\circ} \mathrm{C}$ (bath heating rate of $0.5{ }^{\circ} \mathrm{C} / \mathrm{min}$ ) from $25^{\circ}$ to $45{ }^{\circ} \mathrm{C}$. Each temperature change was followed by a waiting period of at least one hour with the count rate closely monitored in order for the sample to equilibrate. After an hour of waiting (including 20-min of stable count rate) three to four identical experiments were performed at a given temperature. The duration of each experiment was at least $30 \mathrm{~min}$. In other words, the time separation between two subsequent $5{ }^{\circ} \mathrm{C}$ temperature changes was on average equal to three and half hours.

Under the slow heating protocol the waiting times, the number of repeated runs, and the duration of each experiment were largely kept the same as for the fast heating. The only difference between the two heating protocols was the size of the temperature step used. Starting at $39^{\circ} \mathrm{C}$ the heating step of the slow heating was reduced to $0.5^{\circ} \mathrm{C}$ (bath heating rate of $0.16^{\circ} \mathrm{C} / \mathrm{min}$ ). The new heating step was introduced only at $39{ }^{\circ} \mathrm{C}$ since no measurable effect of different heating rate on microgel size distribution was observed below this temperature. This is consistent with observations of uncrosslinked polymer in ref. 16 . The highest temperature reproducibly studied under the slow heating protocol was $42.5^{\circ}$. At higher temperatures, the count rate was fluctuating by more than $25 \%$ and visual inspection of solution revealed big aggregates that, with time, precipitated by gravity.

\section{RESULTS AND DISCUSSION}

Figure 1 shows the observed microgel spectra and the corresponding decay rate distributions both below and above the volume phase transition temperature. The difference between the spectra at the two high temperatures $\left(42^{\circ}\right.$ and 45 ${ }^{\circ} \mathrm{C}$ ) results from the heating history (rate of heating) of the sample: $42{ }^{\circ} \mathrm{C}$ was reached through the 


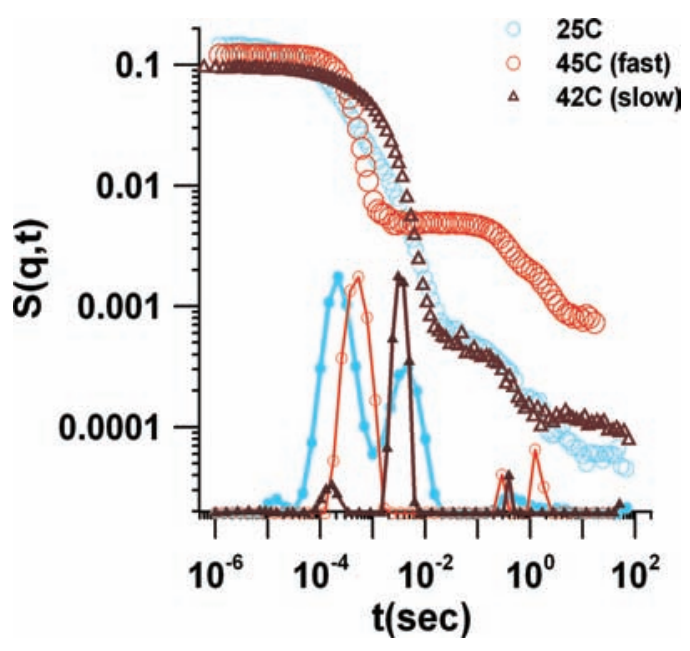

Figure 1. Overlay graph of the intensity correlation functions and corresponding decay time distributions below $\left(25{ }^{\circ} \mathrm{C}\right.$, blue circles) and above the volume phase transition after the fast heating $\left(45^{\circ} \mathrm{C}\right.$, red circles $)$ and after the slow heating $\left(42{ }^{\circ} \mathrm{C}\right.$, brown triangles $)$.

slow heating and $45^{\circ} \mathrm{C}$ through the fast heating processes described in detail in previous section. The decay time distributions here are obtained by the Inverse Laplace Transform algorithm called CONTIN $^{32}$ (ALV5000 version). The shape of the spectrum and the corresponding decay time distribution below the volume phase transition (at $25{ }^{\circ} \mathrm{C}$ ) differ considerably from the two spectra and distributions above the transition. Moreover, the intensity correlation functions and the corresponding decay time distributions at the final temperatures $\left(42^{\circ}\right.$ and $\left.45^{\circ} \mathrm{C}\right)$ of the two heating protocols are very different.

The details of the fast heating transition from $25^{\circ}$ to $45{ }^{\circ} \mathrm{C}$ have been described in ref. 28 . The main finding was that at $t \leq 50 \mathrm{~ms}$ the fast heating resulted in a transition from an apparently bimodal decay time distribution with peaks at 0.2 $\mathrm{ms}$ and $4.6 \mathrm{~ms}$ at $25{ }^{\circ} \mathrm{C}$ to an unimodal distribution centered around $0.5 \mathrm{~ms}$ at $45^{\circ} \mathrm{C}$ (see blue and red distributions on Figure 1). The corresponding apparent radius distribution of microgels (for $t \leq 50 \mathrm{~ms}$ ) switches from the broad distribution that covers the range of 1 to $1700 \mathrm{~nm}$ with two peaks at 30 and $550 \mathrm{~nm}$ to the narrower distribution centered at $150 \mathrm{~nm}$ and ranging between 25 and $400 \mathrm{~nm}$.

On the other hand, the slow heating resulted in a transition from the same wide bimodal decay time distribution at $25{ }^{\circ} \mathrm{C}$ to another bimodal decay time distribution which is only slightly narrower but has one clearly dominant peak at $\approx 3$ $\mathrm{ms}$. The corresponding microgel size distribution (at $t \leq 50 \mathrm{~ms}$ ) after the slow heating (at $42{ }^{\circ} \mathrm{C}$ ) is wide and ranges from to 1 to $1000 \mathrm{~nm}$ with a dominant peak at $590 \mathrm{~nm}$ and a second smaller peak at $30 \mathrm{~nm}$. In other words, the slow heating produced a somewhat wider size distribution of considerably larger microgels than the fast heating of the same starting sample.

The microgel spectra below and above the volume phase transition were systematically and reproducibly characterized using the time moment analysis ${ }^{22,23}$ described in the previous section. $g^{(1)}(q, t)$ obtained from the spectra was fitted to a sum of three stretched exponentials $(i=1,2,3)$ with nine fitting parameters according to eq 2. Each of the three modes is described by an amplitude $\left(A_{i}\right)$, a pseudorate of relaxation $\left(\theta_{i}\right)$, and a stretching parameter $\left(\beta_{i}\right)$. The modes are labeled according to the apparent values of calculated decay rate $\left\langle\Gamma_{i}\right\rangle$ so that the first mode is "fast" with $5 \times 10^{3} \leq\left\langle\Gamma_{1}\right\rangle \leq 10^{4} \mathrm{~s}^{-1}$; the second mode is "intermediate" with $2 \times 10^{2} \leq\left\langle\Gamma_{2}\right\rangle \leq 10^{3} \mathrm{~s}^{-1}$; the third mode is "slow" with $6 \times 10^{-2} \leq\left\langle\Gamma_{3}\right\rangle \leq 4 \mathrm{~s}^{-1}$.

Figures 2-4 analyze the behavior of nine fitting parameters of the field correlation function under the slow heating process from the ambient conditions to temperatures above the volume phase transition.

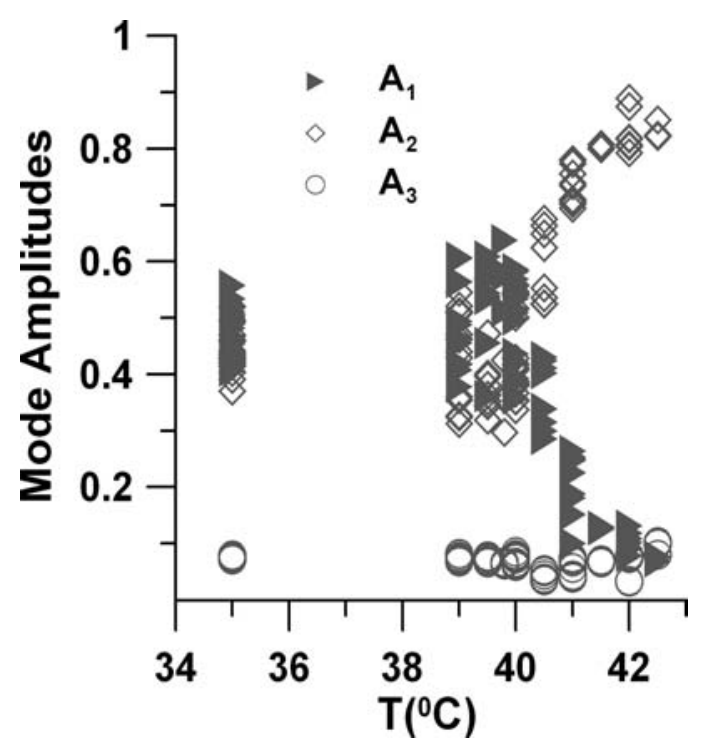

Figure 2. Relative amplitudes of the HPC microgel spectral modes as a function of temperature under the slow heating protocol: the "fast" mode, $A_{1}$ (triangles); the "intermediate" mode, $A_{2}$ (diamonds); the "slow" mode, $A_{3}$ (circles). 


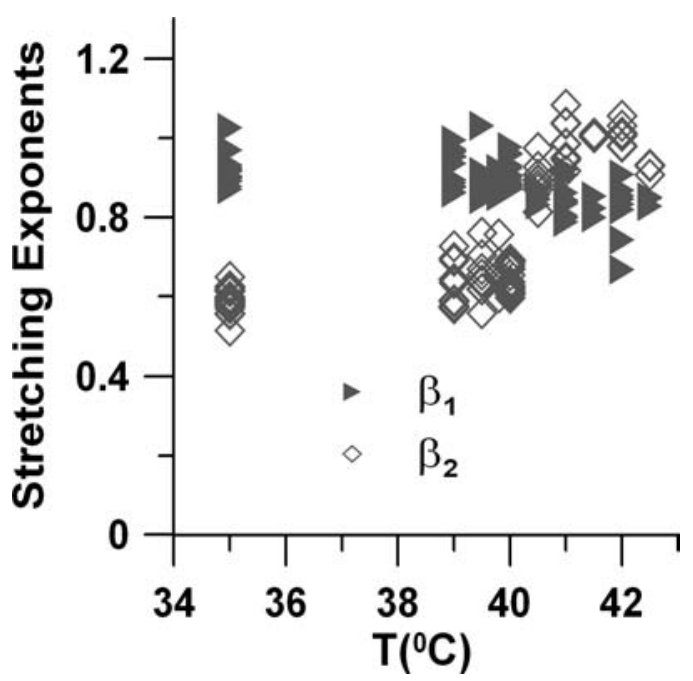

Figure 3. The stretching exponents of the "fast" $\left(\beta_{1}\right.$, triangles) and "intermediate" ( $\beta_{2}$, diamonds) spectral modes of HPC microgel as a function of solution temperature under the slow heating protocol. $\beta_{3}$ (not shown here for clarity) is strongly scattered between 0.5 and 1.05 and shows no apparent temperature dependence.

Figure 2 shows the temperature dependence of the microgel mode amplitudes at $35^{\circ} \leq T \leq 42.5$ ${ }^{\circ} \mathrm{C}$ under the slow heating protocol. Similarly to the mode amplitude behavior (at $T \leq 40{ }^{\circ} \mathrm{C}$ ) under the fast heating protocol (Figure 4 in ref. 28 ), the amplitudes of the "fast" and "intermediate" modes ( $A_{1}$ and $A_{2}$, respectively) under the slow heating have values between 40 and $60 \%$ with the faster mode being slightly more dominant $\left(A_{1} \geq A_{2}\right)$. The third ("slow") mode $\left(A_{3}\right)$ is represented only by about $10 \%$ as was the case under the fast heating. At $T \geq 40{ }^{\circ} \mathrm{C}$, however, the fast heating protocol results in a somewhat different microgel mode distribution than the one obtained under the slow heating. Indeed, the contribution of the "slow" mode $\left(A_{3}\right)$ under the slow heating was not larger above the transition as in the case of the fast heating. Also, the amplitude of the "fast" mode $\left(A_{1}\right)$ shows a steady decrease, while the amplitude of the "intermediate" mode $\left(A_{2}\right)$ reveals a steady increase as temperature rises above $40{ }^{\circ} \mathrm{C}$. Reference 28 reported that $A_{2} \approx 0$ at $45{ }^{\circ} \mathrm{C}$ under the fast heating process and not $A_{2}>0.5$ as seen on Figure 2. This result is, nevertheless, consistent with ref. 28 since under the fast heating there is only one measured temperature above $40^{\circ} \mathrm{C}$ (namely $45^{\circ} \mathrm{C}$ ) and the two modes ("fast" and "intermediate") converge at that temperature. Therefore, at $45{ }^{\circ} \mathrm{C}$ it is impossible to distinguish the two modes after the fast heating as they have the same apparent decay rates.

The transition in the mode character above 40 ${ }^{\circ} \mathrm{C}$ is also seen on the graph of stretching exponents for the two faster microgel modes (Figure 3 ). Indeed, the "fast" mode has a temperatureindependent $\beta_{1} \approx 0.85-1.05$ below $40{ }^{\circ} \mathrm{C}$ which slightly decreases above $40{ }^{\circ} \mathrm{C}$. The "intermediate" mode shows an apparent abrupt transition from weakly temperature dependent $0.5 \leq \beta_{2} \leq 0.7$ below $40{ }^{\circ} \mathrm{C}$ to a temperature independent $0.8 \leq$ $\beta_{2} \leq 1.05$ above $40{ }^{\circ} \mathrm{C}$. The slow mode stretching exponent $\beta_{3}$ (not shown on Figure 3 ) is very scattered and ranges between 0.5 and 1.05.

Figure 4 demonstrates all three pseudorates of relaxation, not only for microgel modes $\left(\theta_{1}, \theta_{2}\right)$, but also for the mode identified by ref. 28 as loose polymer mode $\left(\theta_{3}\right) . \theta_{1}$ under the slow heating appears to be temperature independent and centered around $1.5 \times 10^{-4}(1 / \mathrm{sec})^{\beta} . \theta_{2} \approx 8 \times 10^{-4}-2 \times$ $10^{-3}(1 / \mathrm{sec})^{\beta}$ is also temperature independent up to $40{ }^{\circ} \mathrm{C}$ but increases by a factor of three or four with the temperature increase from $40^{\circ}$ to $42.5^{\circ} \mathrm{C}$. This temperature dependence of $\theta_{2}$ under the slow heating is different from $\theta_{2}$ behavior under the fast heating, ${ }^{28}$ where the pseudorate decreased by a factor of four with the rise of the temperature from $25^{\circ}$ to $45^{\circ} \mathrm{C}$. On the other hand, the dependence of $\theta_{3}$ on temperature seen on Figure 4 is very similar

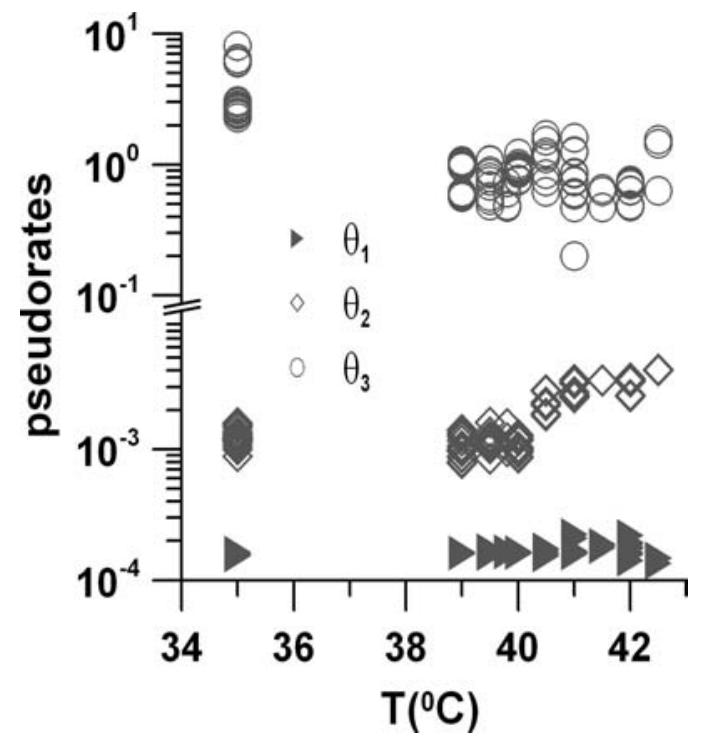

Figure 4. The pseudorates of relaxation (see eq 2) for the "fast", $\theta_{1}$, (triangles), "intermediate", $\theta_{2}$, (diamonds), and "slow" $\theta_{3}$, (circles) spectral modes of HPC microgel as a function of solution temperature under the slow heating protocol. 


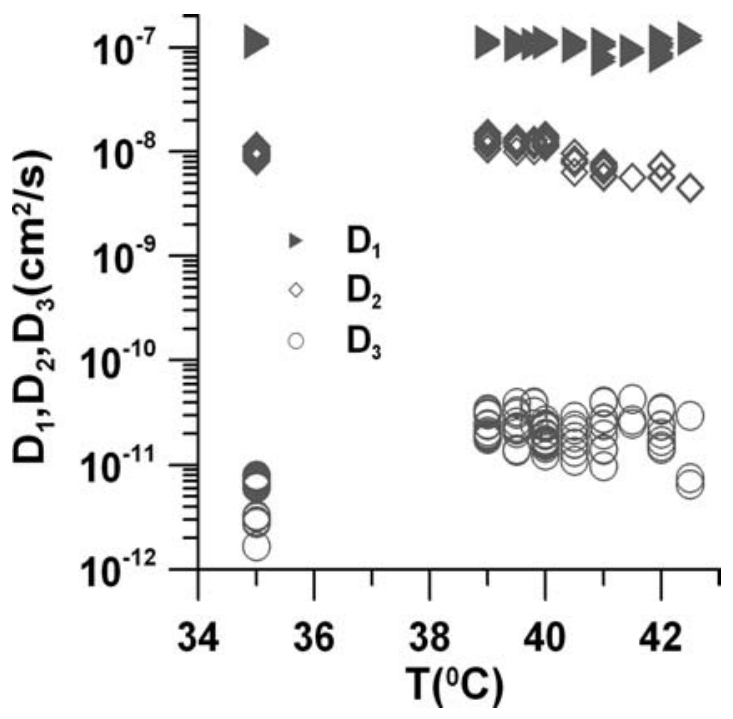

Figure 5. The temperature dependence of the mean diffusion coefficients obtained from the spectral time moment analysis ${ }^{23}$ for each of the observed HPC microgel modes: "fast" (triangles), "intermediate" (diamonds), and "slow" (circles). The slow heating protocol was used.

to the behavior observed for this mode pseudorate under the fast heating. ${ }^{28}$ In both cases, the values of $\theta_{3}$ are strongly scattered around $10^{0}(1 / \mathrm{sec})^{\beta}$ and do not have a clearly identifiable temperature dependence (though $\theta_{3}$ at $35{ }^{\circ} \mathrm{C}$ is somewhat higher than $\theta_{3}$ at other temperatures).

The obtained fitting parameters $\theta_{i}$ and $\beta_{i}$ were used to calculate zeroth time moments $M_{0 i}$ for each mode using eq. 3 which, in turn, yielded mean decay rate $\left\langle\Gamma_{i}\right\rangle$ and mean diffusion coefficient $D_{i}$ for each mode. Figure 5 shows the diffusion coefficients obtained this way for all three modes. The "fast" microgel mode reveals a temperature independent $8 \times 10^{-8} \leq D_{1} \leq 1.5 \times 10^{-7} \mathrm{~cm}^{2} / \mathrm{s}$. The "intermediate" microgel mode shows a transition at $40{ }^{\circ} \mathrm{C}$ from temperature-independent $D_{2} \approx 10^{-8}$ $\mathrm{cm}^{2} / \mathrm{s}$ to $D_{2}$ that decreases from $10^{-8}$ to $4.5 \times 10^{-9}$ $\mathrm{cm}^{2} / \mathrm{s}$ with temperature increase from $40^{\circ}$ to 42.5 ${ }^{\circ} \mathrm{C}$. The "slow" mode (attributed to loose polymer in solution) has a largely temperature-independent and somewhat scattered values of $D_{3}$ ranging from $2 \times 10^{-12}$ to $4 \times 10^{-11} \mathrm{~cm}^{2} / \mathrm{s}$. The values and the temperature dependence of $D_{1}$ obtained under the slow heating are similar to the values and $D_{1}(T)$ obtained under the fast heating protocol. The behavior of $D_{2}$ is, however, different between the two heating protocols. In contrast to $D_{2}(T)$ under the slow heating protocol, where $D_{2}$ decreases by a factor of two above $40{ }^{\circ} \mathrm{C}, D_{2}$ under the fast heating increased by more than five-fold from about
$4 \times 10^{-9}$ to $2.5 \times 10^{-9}$ as the temperature was raised from $25^{\circ}$ to $45^{\circ} \mathrm{C} .{ }^{28}$ Finally, the values and the lack of temperature dependence for $D_{3}$ under the slow heating is identical to the values and the behavior of $D_{3}(T)$ under the fast heating protocol.

The importance of the heating rate on microgel volume phase transition is summarized by Figure 6 which compares apparent hydrodynamic radii for the "fast" and "intermediate" modes under the fast and slow heating. The "fast" microgel mode hydrodynamic radius $R_{\mathrm{H} 1} \approx 25-40 \mathrm{~nm}$, obtained under the slow heating, is consistent with $R_{\mathrm{H} 1}$ obtained under the fast heating at $T \leq 40{ }^{\circ} \mathrm{C}$. Above $40{ }^{\circ} \mathrm{C}, R_{\mathrm{H} 1}$ from the slow heating is somewhat scattered, but does not change significantly in magnitude for the range of temperatures studied. $R_{\mathrm{H} 1}$ from the fast heating, on the other hand, increases up to four orders of magnitude as temperature changes from $40^{\circ}$ to $45^{\circ} \mathrm{C}$. The values of hydrodynamic radius $R_{\mathrm{H} 2}(\approx 2500-650 \mathrm{~nm})$ corresponding to the intermediate microgel mode for the two different heating protocols are also similar at $T \leq 40{ }^{\circ} \mathrm{C}$. In this temperature range, the shrinking in $R_{\mathrm{H} 2}$, which is apparent under the fast heating, is consistent with the four data

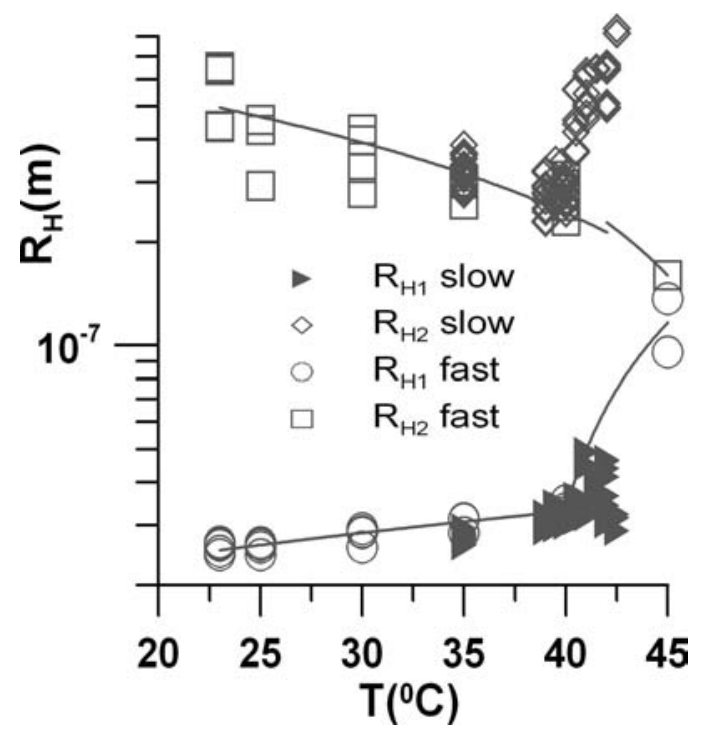

Figure 6. The comparison of the apparent hydrodynamic radii $R_{\mathrm{Hi}}$ for the two diffusive microgel modes under the fast and slow heating protocols. The "fast" modes (triangles for the slow and circles for the fast heating) correspond to $\left\langle\Gamma_{1}\right\rangle \approx 2-8 \times 10^{3} 1 / \mathrm{s}$. The "intermediate" modes (diamonds for the slow heating and squares for the fast heating) correspond to $\left\langle\Gamma_{2}\right\rangle \approx$ $300-7001 / \mathrm{s}$. The lines are guides for the eye. The fast heating data comes from ref. 28. 


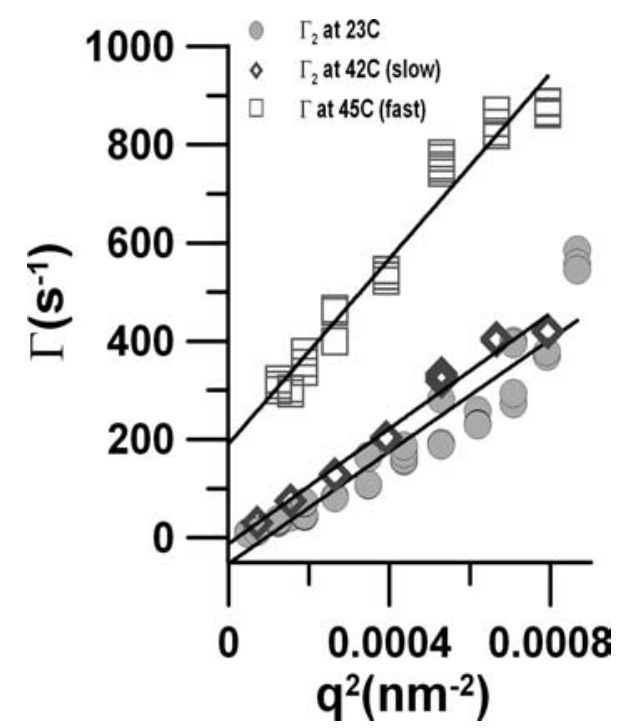

Figure 7. Mean decay rates for the dominant spectral mode ("intermediate" for $23^{\circ}$ and $42{ }^{\circ} \mathrm{C}$ ) of HPC microgel as a function scattering wave vector squared. $\Gamma_{2}(q)$ of microgels at $23{ }^{\circ} \mathrm{C}$ (filled circles) is compared to $\Gamma_{2}(q)$ of microgels after the slow heating (diamonds, at $42{ }^{\circ} \mathrm{C}$ ) and $\Gamma(q)$ of microgels after the fast heating (squares, at $45^{\circ} \mathrm{C}$ ). The lines are linear fits to the data. The $23{ }^{\circ} \mathrm{C}$ data is borrowed from ref. 28.

points obtained under the slow heating at $35^{\circ} \leq$ $T \leq 40{ }^{\circ} \mathrm{C}$. However, above $40^{\circ} \mathrm{C}$ the slow heating reverses the trend and brings a three-fold increase in $R_{\mathrm{H} 2}$ (up to $800-850 \mathrm{~nm}$ ) as temperature rises to $42.5^{\circ}$. This result comes in direct contrast to the result from the fast heating, where the hydrodynamic radius of the "intermediate" mode shrinks down to less than $200 \mathrm{~nm}$ above the transition. ${ }^{28}$ This finding confirms the hypothesis that the HPC microgel's volume phase transition depends on the heating history of the sample. Indeed, the observed difference between $R_{\mathrm{H} 2}$ under two different heating protocols is dramatic.

Finally, Figure 7 shows the $q$-dependences of the mean relaxation rates for the dominant microgel mode below and above the transition. The angular dependence of the mean relaxation rate $\Gamma_{2}$ for the "intermediate" mode at $23^{\circ} \mathrm{C}$ (data from ref. 28 ) is compared to the $q$-dependences of the corresponding $\Gamma$ after the slow heating (at $42{ }^{\circ} \mathrm{C}$ ) and the fast heating (at $45^{\circ} \mathrm{C}$ ). The choice of the relaxation rates compared on this figure is dictated by the mode dominance at $42^{\circ}$ and $45{ }^{\circ} \mathrm{C}$. Indeed, Figure 2 shows that the amplitude of the "intermediate" mode at $42{ }^{\circ} \mathrm{C}$ is about $80-90 \%$, while ref. 28 reported the corresponding mode amplitude to be about $65-80 \%$. At room temperature $\left(23{ }^{\circ} \mathrm{C}\right)$, the contribution of this mode is $q$-dependent and varies between $40 \%\left(\right.$ at $\theta=130^{\circ}$ ) and $75 \%$ (at $\theta=$ $25^{\circ}$ ). The $q$-dependence of this mode reveals diffusive like behavior $\left(\Gamma(q) \sim q^{2}\right)$ at three extreme temperatures studied. In particular, $\Gamma_{2}$ for the 42 ${ }^{\circ} \mathrm{C}$ sample increases linearly with $q^{2}$ with negligible intercept at $q=0 . \Gamma_{2}(q)$ for the microgels at room temperature is somewhat scattered, but also is linear in $q^{2}$ and has a small negative intercept (which might be due to the data scatter). The $q$-dependence of $\Gamma$ for $45{ }^{\circ} \mathrm{C}$ sample is also linear in $q^{2}$, but has a significant positive intercept. Figure 7 also confirms that the dominant microgel mode after the slow heating corresponds to the particulates of the size comparable to the microgel size of the "intermediate" mode at $23{ }^{\circ} \mathrm{C}$ but 2-2.5 times larger than the de-swollen microgels after the fast heating. The $q$-dependences of the other microgel modes and their amplitudes appear elsewhere. ${ }^{28,37}$

\section{CONCLUSIONS}

The dynamic light scattering data on HPC microgels synthesized in a surfactant-free addition reaction (following the recipe of ref. 17) was carefully studied at different temperatures and scattering angles by ref. 28. Observed highly nonexponential spectra of microgels were analyzed with the line shape analysis and spectral time-moments method ${ }^{22,23}$ instead of relying on cumulant expansion, which can cause serious misinterpretation of nonexponential, multimodal DLS spectra ${ }^{13}$ or CONTIN, which is known to be highly sensitive to experimental noise in systems with long relaxation times. The line shape analysis revealed that the synthesized microgels are multimodal. ${ }^{28}$ At ambient conditions, two of the three observed spectral modes show diffusive-like properties, which likely correspond to particulates with diffusion coefficients of $\approx 10^{-7}$ and $\approx 10^{-8} \mathrm{~cm}^{2} / \mathrm{s}$ and apparent hydrodynamic radii of 25-30 $\mathrm{nm}$ and 400-650 $\mathrm{nm}$. Above the volume phase transition resulting from the fast (one step) heating, the two modes collapse into one with diffusion coefficient of $2-3 \times$ $10^{-8} \mathrm{~cm}^{2} / \mathrm{s}$ and an apparent $R_{\mathrm{H}}$ of $100-150 \mathrm{~nm}$. The third observed mode was found ${ }^{28}$ to have properties (temperature and angular dependencies) of uncrosslinked polymer chains and has been attributed to loose polymer chains in microgel solution. 
This study expanded the findings of ref. 28 by showing that the rate of heating of the microgel solution dramatically affects the volume phase transition of microgels. During the fast (one step) heating from $40^{\circ}$ to $45{ }^{\circ} \mathrm{C}$ a significant reduction in the width of microgel size distribution has been observed with the "fast" mode disappearing and the "intermediate" mode revealing a two- to threefold shrinkage of apparent hydrodynamic radius. However, the slow (multistep) heating from $40^{\circ}$ to $42.5{ }^{\circ} \mathrm{C}$ has a very different effect on the two particulate microgel modes. The modes do not collapse into one. Instead, the "fast mode" is still present at the highest $T$ even though its amplitude is reduced significantly from $40-60$ to $10 \%$. Particulates of this mode exhibit fast diffusion $\left(10^{-7} \mathrm{~cm}^{2} / \mathrm{s}\right)$ and an apparent size of $20-40 \mathrm{~nm}$. The "intermediate mode" under the slow heating shows temperature dependence similar to the one under the fast heating but only up to 40 ${ }^{\circ} \mathrm{C}$. Indeed, under both heating protocols microgels of "intermediate" mode deswell by a factor of two in size as $T$ increases from 25 to $40{ }^{\circ} \mathrm{C}$. However, above $40{ }^{\circ} \mathrm{C}$, microgels of the "intermediate" mode under the slow heating start to grow instead of shrinking in size. They grow from 200$300 \mathrm{~nm}$ to $800-850 \mathrm{~nm}$ as $T$ is increased in a multistep process from 40 to $42.5{ }^{\circ} \mathrm{C}$. The contribution of this "intermediate" mode increases up to 80-90\%. Apparently, the long incubation of microgels at each temperature above $40{ }^{\circ} \mathrm{C}$ gives more time for uncrosslinked polymer chains as well as smaller crosslinked aggregates to associate with "intermediate" mode microgels to form even larger HPC aggregates. According to the angular dependence results (Figure 7), these aggregates exhibit purely diffusive behavior with $\Gamma_{2} \sim q^{2}$ over wide range of angles with negligible intercept. In fact, it appears that the HPC aggregates after the slow heating have more diffusive-like behavior than their counterparts at ambient conditions. At the temperatures higher than $42.5^{\circ} \mathrm{C}$ precipitation of the polymer aggregates by gravity was observed.

The dependence of the microgel volume phase transition on the heating history mimics the dependence of the formation of metastable polymer aggregates above $T_{\mathrm{c}}$ in uncrosslinked HPC solutions as observed by Gao et al. ${ }^{16}$ Reference 16 found that the multistep heating of uncrosslinked HPC solution to $42{ }^{\circ} \mathrm{C}$ resulted in metastable aggregates that had a $35 \%$ larger apparent $R_{\mathrm{H}}$ than the aggregates obtained under one step heating from $25^{\circ}$ to $42{ }^{\circ} \mathrm{C}$. Reference 16 also reported the growth of HPC aggregates as temperature was raised above $42^{\circ}$. The growth of metastable HPC formations continues up to a specific cutoff temperature (about $50{ }^{\circ} \mathrm{C}$ ) at which the precipitation of polymer aggregates by gravity was observed. Similarly, Carotenuto et al. ${ }^{35}$ who studied thermo reversible gelation of HPC aqueous solutions, suggested that the observed phase transition consisted of two steps: precipitation followed by sol-gel transition. They discovered that the heating rate affects the twostep process because weaker gels were formed at lower heating rates as more precipitation takes place and fewer "active" chains (chains present in solution after precipitation) were available for gelation.

The findings of this article on the effect of the heating rate on the size of HPC microgel particles agree with the findings of ref. 16 on uncrosslinked polymer. In both cases, the multistep slow heating above the critical temperature resulted in larger particles than the ones obtained under the one step fast heating. It is a somewhat surprising finding for microgels as we expected them to deswell upon heating. It is also an important finding that suggests that in multimodal microgel systems (synthesized following the recipe of ref. 17) the presence of uncrosslinked HPC chains and smaller crosslinked HPC aggregates (with size of 25-30 nm) influences the volume phase transition of the main microgel mode (with the size of $400-650 \mathrm{~nm}$ ). This conclusion is consistent with the findings of ref. 35 on the effect of the heating rate on the gelation process.

The conclusions of this article need to be further validated by additional experiments. Work is underway ${ }^{37}$ to study the effects of salt and polymer concentration, amount of crosslinker and synthesis techniques on the volume phase transition in HPC microgel system. The new results should help to outline the mechanism of the volume phase transition in HPC microgels as well as to shed more light on the processes of phase transition and gelation in uncrosslinked HPC solutions.

The authors acknowledge R. Mohieddine for prepared samples of crosslinked HPC and K. Freeman for help with $q$-dependence measurements at $42^{\circ} \mathrm{C}$. The authors are also deeply thankful to R. McDonough for useful discussions and paper proofreading. This work was supported by the Cottrell Science Award from the Research Corporation and the Engaged Learning Undergraduate Student Award from CSU. 


\section{REFERENCES AND NOTES}

1. Pelton, R. Adv Colloid Interface Sci 2000, 85, 1-33.

2. Murray, M. J.; Snowden, M. J. Adv Colloid Interface Sci 1995, 54, 73-91.

3. Saunders, B. R.; Vincent, B. Adv Colloid Interface Sci 1999, 80, 1-25.

4. Lu, X.; Hu, Z.; Gao, J. Macromolecules 2000, 33, 8698-8702.

5. Langevin, D. Annu Rev Phys Chem 1992, 43, 341-352.

6. Ngai, T.; Auweter, H.; Behrens, S. H. Macromolecules $2006,8171-8177$.

7. Oh, J. K.; Drumright, R.; Siegwart, D. J.; Matyjaszewski, K. Prog Polym Sci 2008, 33, 448-477.

8. Snowden, M. J.; Vincent, B.; Morgan, J. C. UK Patent GB 226, 2,117,A 1993; (b) Li, Y.; Liu, Y.; Bai, B. Oil Drilling Prod Technol 1999, 21, 3.

9. Jones, C. D.; Lyan, L. A. J. Am Chem Soc 2003, $125,460-465$

10. Su, S.; Ali, M. M.; Filipe; C. D. M.; Li, Y.; Pelton, R. Biomacromolecules 2008, 9, 935-941; (b) Freemont, T. J.; Saunders, B. R. Soft Matter 2008, 4, 919-924.

11. Wu, C. Polymer, 1998, 39, 4609-4619; (b) Peng, S.; Selser, J. C.; Bogoslovov, R.; Piet, G. J Chem Phys 2004, 120, 8841-8852.

12. Aharoni, S. M. Macromolecules 1983, 16, 1722 1728.

13. Mustafa, M.; Russo, P. S. J Colloid Interface Sci 1989, 129, 240-245.

14. Werbowyj. R. S.; Gray. D. G. Macromolecules 1980, $13,69-73$.

15. Russo, P. S.; Mustafa, M.; Cao, T.; Stephens, L. K. J Colloid Interface Sci 1988, 122, 120-137.

16. Gao, J.; Haidar; G.; Lu, X.; Hu, Z. Macromolecules 2001, 34, 2242-2247.

17. Xia, X.; Tang, S.; Lu, X.; Hu, Z. Macromolecules 2003, 36, 3695-3698.

18. Mustafa, M. B.; Tipton, D. L.; Barkley, M. D.; Russo, P.S.; Blum, F. D. Macromolecules 1993, 26, 370-378.

19. Guido, S. Macromolecules 1995, 28, 4530-4539.

20. Yang, T.; Jamieson, A. M. J. Colloid Interface Sci 1988, 126, 220-230; (b) Brown, W.; Rymden, R. Macromolecules 1986, 19, 2942-2952; (c) Bu, Z; Russo, P. S. Macromolecules 1994, 27, 1187-1194; (d) Phillies, G. D. J.; Quinlan, C. A. Macromolecules 1995, 28, 160-164; (e) Phillies, G. D. J.; LaCroix, M. J. Phys Chem B 1997, 101, 39-47; (f) Streletzky, K. A.; Phillies, G. D. J. J Chem
Phys 1998, 108, 2975-2988; (g) Streletzky, K. A.; Phillies, G. D. J. J. Polym Sci Part B: Polym Phys 1998, 36, 3087-3099; (h) Streletzky, K. A.; Phillies, G. D. J. Macromolecules 1999, 32, 145-152; (i) Streletzky, K. A.; Phillies, G. D. J. J Phys Chem B 1999, 103, 1811-1820; (j) O'Connell, R. O., Hanson, H., Phillies, G. D. J. J Polym Sci Part B: Polym Phys 2004, 43, 323-333.

21. Phillies, G. D. J.; Richardson, C.; Quinlan, C. A.; Ren, S. Z. Macromolecules 1993, 26, 6849 6858.

22. Phillies, G. D. J.; O'Connell, R.; Whitford, P.; Streletzky, K. A. J Chem Phys 2003, 119, 99039913.

23. Phillies, G. D. J.; Streletzky, K. A. In Soft Condensed Matter: New Research; Dillon, K. Ed.; Nova Science: New York, 2007, pp 291-335.

24. Guo, J.-H.; Skinner, G. W.; Harcum, W. W.; Barnum, P. E. PSTT 1998, 1, 254-261.

25. Brydon, G.; Ganguly, R.; Ghosh, S. Gut 2003 52, A9; (b) Watano, S.; Nakamura, H.; Hamada, K.; Wakamatsu, Y.; Tanabe, Y.; Dave, R. N.; Pfeffer, R. Powder Technol 2004, 141, 172-176.

26. Machida, Y.; Nagai, T. Chem Pharm Bull 1974, 22, 2346-2351.

27. Skinner, G. W.; Harcum, W. W.; Barnum, J. H. Guo J. H. Drug Dev Ind Pharm 1999, 25, 1121-1128.

28. Streletzky K. A.; McKenna, J. T.; Mohieddine, R. J Polym Sci Polym B Polym Phys 2008, 46, 771-781.

29. Cai, T.; Hu, Z. Macromolecules 2003, 36, 6559 6564.

30. Francis, M.; Piredda, M.; Winnik, F. J Controlled Release 2003, 93, 59-68.

31. Koppel, D. E. J Chem Phys 1972, 57, 4814-4820.

32. Provincher, S. W. Macromol Chem 1979, 180, 201215.

33. Phillies, G. D. J.; Streletzky, K. A. Recent Res Dev Phys Chem 2001, 5, 269-285.

34. (a) Kyu, T.; Mukhejee, P. Liq Cryst 1988, 3, 631644; (b) Kyu, T.; Zhuang, P.; Mukhejee, P. In Polymer Association Structures. Microemulsions and Liquid Crystals; ACS Symposium Series ElNokaly, M. A., Ed.; ACS: Washington, DC, 1989, 384 , pp 266-283.

35. Carotenuto, C.; Grizzuti, N. Rheo Acta 2006, 45, 468-473.

36. Altenberger, A. R.; Deutch, J. M. J Chem Phys 1973, 59, 894-898.

37. Streletzky, K. A.; Benmezouga, I.; McKenna, J. T., to be submitted. 